\title{
Avaliação de desempenho e consumo de energia de um ambiente virtual de aprendizagem em nuvens privadas
}

\section{Performance evaluation and energy consumption of a virtual learning environment on private clouds}

\author{
Claudemir Jeremias de Lima ${ }^{\mathscr{E}, 1}$, Alison Vinicius Gomes da Silva ${ }^{1}$, Ermeson \\ Carneiro de Andrade ${ }^{1}$, Gustavo Rau de Almeida Callou ${ }^{(\mathbb{}, 1}$ \\ ${ }^{1}$ Departamento de Computação, Universidade Federal Rural de Pernambuco \\ *claudemirjlima@ufrpe.br; alison.gsilva@ufrpe.br; ermeson.andrade@ufrpe.br; gustavo.callou@ufrpe.br
}

Recebido: 14/07/2020. Revisado: 05/03/2021. Aceito: 29/03/2021.

\begin{abstract}
Resumo
Os Ambientes Virtuais de Aprendizagem (AVA) estão cada vez mais presentes nas instituições de ensino, visto que consistem em mais um recurso para melhorar a comunicação entre alunos e professores. Embora esses sistemas não sejam novos, poucos estudos têm avaliado a performance e o consumo de energia dos mesmos. Avaliar a relação entre a performance e o consumo de energia dos AVAs é essencial para solucionar questões tanto na fase de implementação de um novo projeto quanto na ampliação ou atualização da infraestrutura já existente. Portanto, este trabalho visa avaliar o desempenho e o consumo de energia do ambiente MOODLE, plataforma que fornece suporte a AVAs, utilizando dois sistemas de nuvens privadas, o Apache CloudStack e OpenStack. Uma metodologia é proposta para proporcionar a realização de tais experimentos através de medições. Um estudo de caso foi realizado para ilustrar a aplicabilidade da metodologia em um ambiente real com o Moodle configurado tanto no Apache CloudStack quanto no OpenStack. Os resultados revelaram que o desempenho e também o consumo de energia são diferentes entre os dois sistemas. Por exemplo, nos experimentos realizados, o OpenStack apresentou um maior consumo de energia quando comparando ao Apache CloudStack por demandar um maior tempo de resposta.
\end{abstract}

Palavras-Chave: Computação em Nuvem; Avaliação de Desempenho; Consumo de Energia; Ambiente Virtual de Aprendizagem.

\section{Abstract}

Virtual Learning Environments (VLE) are increasingly present in educational institutions and represents a new resource that is able to improve the communication between students and teachers. Although these systems are not new, few studies are available for the integrated evaluation of their performance and energy consumption. Assessing the relationship between the performance and energy consumption of VLEs is essential to solving issues both in the implementation phase of a new project and in the expansion or updating of a previous existing infrastructure. Therefore, this work aims to evaluate the performance as well as the energy consumption of the MOODLE environment, a platform that supports VLEs, through two private cloud systems, Apache CloudStack and OpenStack. A methodology is proposed to allow one to conduct such experiments through measurements. A case study illustrates the applicability of the proposed methodology, assuming the Moodle configured on both Apache CloudStack and OpenStack. The results revealed the performance, and also the energy consumption, are different between the two systems, where OpenStack showed a higher energy consumption when compared to Apache CloudStack due to its higher demands on response time.

Keywords: Cloud Computing; Performance Evaluation; Energy Consumption; Virtual Learning Environment. 


\section{Introdução}

A computação em nuvem está cada vez mais presente na sociedade em diversas áreas (ex.: educacional, comercial ou financeira) devido às suas vantagens como alocação dinâmica de recursos e baixo custo. Existem diversos exemplos de serviços de $e$-commerce, plataforma de streaming e ambiente virtuais de aprendizagem que necessitam de recursos dinâmicos e uma alta disponibilidade, que é facilmente provido pelo paradigma de computação em nuvens.

O AVA é uma plataforma colaborativa que é utilizada no ensino a distância, podendo ser utilizada também no ensino presencial, além de um acompanhamento de grupos de trabalho. No entanto, tal ambiente demanda elevado desempenho. O desempenho está relacionado diretamente à infraestrutura de Tecnologia da Informação (TI) que é composta por dispositivos de hardware e software, além das tecnologias para realizar a gestão dos dados e também para permitir a comunicação entre os componentes. Existe também uma demanda para se avaliar de forma integrada o desempenho e o consumo de energia de tais ambientes a fim de não se ter um impacto negativo na sustentabilidade e/ou no custo. As infraestruturas dos sistemas distribuídos envolvem diversos componentes, existindo muitos fatores que podem influenciar tanto no desempenho como no consumo de energia de uma determinada aplicação executada em um sistema em nuvem.

Alguns trabalhos disponíveis na literatura propõe modelos analíticos para avaliar AVAs (Kim et al., 2009, Sousa et al., 2017). Modelos analíticos podem lidar com problemas como a simplificação do sistema modelado e, assim, não representar fielmente o sistema. Outros trabalhos focam na medição do sistema em análise (Feitelson, 2015). A medição é capaz de produzir dados mais confiáveis, uma vez que lida com o sistema real. Porém, algumas métricas podem requerem procedimentos complexos, o que pode demandar muito tempo ou mesmo inviabilizar a medição. Por exemplo, medir a disponibilidade do sistema por um longo período (ex., dois anos), ou ainda, realizar análise de sensibilidade nesse sistema para verificar o impacto na disponibilidade. Todavia, a utilização da técnica de medição pode ser altamente eficaz para comparar tanto o consumo como o desempenho de AVAs hospedados em diferentes nuvens privadas.

Os sistemas AVAs não são novos. No entanto, poucos estudos têm avaliado de forma integrada o desempenho e o consumo de energia desses sistemas. Avaliar a relação entre o desempenho e o consumo de energia dos AVAs é essencial para solucionar questões tanto na fase de implementação de um novo projeto quanto na expansão ou atualização de uma infraestrutura já existente. Portanto, este trabalho visa avaliar o desempenho e o consumo de energia do ambiente MOODLE, plataforma que fornece suporte a AVAs, utilizando dois sistemas de nuvens privadas, o Apache CloudStack e OpenStack. Sendo assim, esta pesquisa faz a proposição de uma estratégia computacional para comparar o desempenho do Ambiente Virtual de Aprendizagem(AVA) em diferentes nuvens privadas. Essa estratégia faz uso de medições para se estimar as métricas de interesse.

$\mathrm{O}$ restante deste trabalho está dividido da seguinte forma. A Seção 2 apresenta os trabalhos relacionados. A
Seção 3 apresenta a fundamentação teórica abordando os seguintes assuntos: computação em nuvem e avaliação de desempenho. A Seção 4 apresenta a metodologia usada no trabalho. A Seção 5 descreve o estudo de caso utilizado para ilustrar a aplicabilidade da estratégia proposta. Por fim, a Seção 6 conclui o trabalho e apresenta os futuros direcionamentos dessa pesquisa.

\section{Trabalho Relacionados}

Para posicionar nosso artigo e indicar suas contribuições, primeiro resumiremos os trabalhos relacionados que foram realizados na área de computação em nuvem. Depois disso, discutimos estudos sobre os AVAs. Por fim, fornecemos uma comparação do nosso trabalho em relação a esses trabalhos em termos de escopo e avaliação.

Nos últimos anos, muitos estudos foram realizados para analisar nuvens computacionais. Em (FÉ, 2017), modelos em SPN foram propostos para auxiliar no planejamento de sistemas de transcodificação de vídeo em nuvem privada e pública. Modelos em SPN foram definidos para se estimar as seguintes métricas: vazão, tempo de resposta e custo. Para validar os resultados obtidos através dos modelos SPN, testes foram realizados no ambiente de nuvem Apache CloudStack, usando o Apache JMeter na máquina cliente para enviar as requisições de transcodificações. De acordo com os resultados, observou-se que o tempo de resposta diminuiu de 30 para 15 segundos, e o custo teve um ajuste em aproximadamente de $300 \%$.

Torres et al. (2016) propuseram modelos em SPN, RBD e CTMC para a realização de uma avaliação integrada das métricas de desempenho e disponibilidade de um serviço de armazenamento em uma nuvem privada, gerenciada pelo sistema Eucalyptus. Os autores utilizaram as ferramentas JMeter e o Apache benchmarking Tool para as medições e, consequentemente, para a validação dos modelos propostos. Tais modelos foram validados através das métricas de vazão e nível de utilização do sistema.

Os autores em Bruschi et al. (2016) apresentaram um estudo de caso com o sistema Apache CloudStack utilizando o hypervisor XenServer e armazenamento dos dados no sistema Openfiler. Foram utilizados testes de desempenho a partir de três diferentes tipos de perfil de instâncias em uma nuvem computacional privada. Analisando a medição do consumo de CPU, disco E/S e Memória. Como resultado, foi observado um alto consumo de disco na camada de armazenamento de dados, em particular na gravação de dados de E/S. Também foi registrado um alto consumo de memória na camada de hypervisor, o que é explicado pelo próprio hypervisor, na alocação de VMs sendo empregadas e usadas no processo.

Vogel et al. (2017) realizaram uma pesquisa com experimentos em instâncias utilizando hypervisor KVM e contêiner, em um sistema de nuvens privadas de IaaS com o sistema Apache CloudStack. Para realizar as medições foi utilizado o NetPIPE, obtendo as seguintes métricas: taxa de transferência e a latência da rede. Também foi utilizado o Iperf que é uma ferramenta de benchmarking, sendo uma ferramenta multi protocolo para avaliar o desempenho de redes. Como resultado, observou-se que as instâncias de nuvem baseadas em KVM apresentaram pequena degra- 
dação do desempenho da rede em relação à taxa de transferência quando comparado com as instâncias baseadas em contêiner.

Barros et al. (2017) propuseram uma análise conjunta de desempenho e consumo de energia através da medição de um sistema de armazenamento de dados distribuídos. Para gerar a carga foi utilizado o Yahoo! Cloud Serving Benchmark (YCSB), possibilitando a análise de cenários variando a quantidade de operações de inserção e a quantidade de nós no cluster do Cassandra. Pelos resultados, verificou-se que o aumento no número de nós causa um aumento na vazão, mas consequentemente, também aumenta o consumo de energia elétrica.

No contexto dos AVAs, estudos foram realizados para avaliar esses ambientes. Em Sousa (2015) foi realizado um planejamento para infraestruturas de nuvens privadas. Modelos e uma técnica de otimização com GRASP foram propostos para otimizar custo e desempenho. Os modelos RBD e SPN propostos são capazes de realizar a estimativa de desempenho e disponibilidade. Nos estudos de caso, a autora usou vários esquemas de configuração de software e hardware para avaliar o impacto das alterações de carga, ocorrências de falha e atividades de recuperação no ambiente de nuvem Eucalyptus. Na validação do modelo de desempenho foi utilizado o AVA Moodle configurado em uma máquina virtual na plataforma Eucalyptus.

Os autores em Sousa et al. (2017) apresentaram uma metodologia que faz uso de diagramas de confiabilidade (RBD) e redes de Petri estocásticas (SPN), aliada a uma abordagem de otimização com GRASP para auxiliar no planejamento de infraestruturas de nuvens privadas. Utilizando o Moodle hospedado em uma máquina virtual na plataforma Eucalyptus. As métricas de interesse de estudo foram a de disponibilidade e a de custo.

Os autores em dos Santos et al. (2020) avaliaram o desempenho dos seguintes serviços: OwnCloud, NextCloud e Pydio. Um estudo de caso foi apresentado com a finalidade de mostrar a aplicabilidade desses softwares rodando em uma nuvem privada de baixo custo. $O$ trabalho teve como foco a quantificação do desempenho da CPU, uso de memória e taxa de escrita em disco. No entanto, não foi o foco dos autores comparar o consumo de energia e nem o desempenho de diferentes nuvens privadas.

Em (da Silva et al., 2019), os autores propuseram uma estratégia baseada em modelo de redes de Petri estocástica para avaliar unicamente o desempenho do ambiente virtual de aprendizagem Moodle hospedado na nuvem privada Apache CloudStack. A ideia principal do trabalho era a de analisar o desempenho do Moodle dentro de um cenário onde alunos de uma universidade, em uma semana de provas, por exemplo, deveriam subir as provas em um mesmo horário no sistema. Esse trabalho realizou experimentos com VMs compostas por 1GB e 2GB de memória RAM, e com um único core de CPU de $1 \mathrm{GHz}$. Com essa configuração, conseguiu-se realizar experimentos levando em consideração somente medições de desempenho com o máximo de 40 usuários simultâneos no sistema. Sendo assim, diferente do trabalho anterior, esse trabalho se propõe a analisar tanto o desempenho como o consumo de energia entre duas nuvens privadas, o OpenStack e o Apache CloudStack. Além disso, cenários com complexidade superior foram adotados, levando em consideração
VMs com uma maior quantidade de memória RAM (4GB) e com superior poder computacional (2 cores de CPU de $2 \mathrm{GHz}$ ). Cenários mais complexos e com um maior número de usuários simultâneos acessando as nuvens privadas foram analisados. Sendo assim, esses trabalhos se diferem no objetivo, nas métricas analisadas e nos estudos de casos realizados.

Diferentemente dos estudos anteriores, este trabalho propõe uma estratégia integrada para avaliação tanto do desempenho como do consumo de energia do AVA hospedado em diferentes infraestruturas de nuvens privadas (ex., Apache CloudStack e OpenStack). A Tabela 1 apresenta uma visão geral dos trabalhos relacionados previamente explicados. Pode-se perceber que não foi encontrado um estudo com uma abordagem integrada de desempenho e consumo de energia de AVAs hospedados em diferentes nuvens privadas. A escolha de se analisar, neste trabalho, o Apache CloudStack e o OpenStack foi pelo fato destes dois sistemas serem os mais utilizados na atualidade.

\section{Fundamentação Teórica}

Esta seção apresenta os conceitos necessários para uma melhor compreensão deste trabalho. Inicialmente, serão apresentados os conceitos sobre computação em nuvem (cloud computing), seguidos por avaliação de desempenho.

\subsection{Computação em Nuvem}

(NIST, 2014) definiu a computação em nuvem como um modelo para acesso ubíquo, conveniente e sob demanda a uma rede compartilhada de recursos computacionais configuráveis (por exemplo, redes, servidores, armazenamento, aplicações e serviços) que podem ser rapidamente adquiridos e liberados com mínimo esforço gerencial ou interação com o provedor de serviços.

A computação em nuvem oferece vantagens como compartilhamento de serviços, sistemas operacionais e aplicativos em rede facilitando o gerenciamento dos modelos de negócio sem se preocupar com detalhes da infraestrutura (Machida et al., 2011).

Os três modelos de serviços em nuvem computacional podem ser classificados de acordo com o seu modelo de negócio (Zota and Petre, 2014):

- Infraestrutura como Serviço (Infrastructure as a Service - IaaS): É um modelo de serviço que fornece recursos de computação como hardware, armazenamento e rede.

- Plataforma como Serviço (Platform as a Service - PaaS): é um modelo de serviço onde o usuário pode utilizar para o desenvolvimento e execução de aplicações.

- Software como Serviço (Software as a Service - SaaS): é um modelo de serviço onde são fornecidos aplicativos executados em uma infraestrutura de nuvem.

A grande disponibilidade de recursos e serviços, além do crescente acesso de usuários à computação em nuvem vêm favorecendo o aumento acelerado de hospedagem em diversas soluções de TI. No entanto, existe uma enorme quantidade de configurações possíveis e, assim, é impor- 
Tabela 1: Visão geral dos trabalhos relacionados.

\begin{tabular}{|c|c|c|c|c|}
\hline Trabalho & Estratégia & Métricas de Desempenho & Sistema em Nuvem & AVA \\
\hline Sousa et al. (2017) & $\begin{array}{l}\text { Medição I } \\
\text { Modelagem }\end{array}$ & Memória, CPU & Eucalyptus & MOODLE \\
\hline FÉ (2017) & $\begin{array}{l}\text { Medição I } \\
\text { Modelagem }\end{array}$ & Vazão, Temp. Resp. Consumo energia & Cloudstack & - \\
\hline Torres et al. (2016) & $\begin{array}{l}\text { Medição I } \\
\text { Modelagem }\end{array}$ & $\begin{array}{l}\text { Vazão, Número de falhas, número de atendimen- } \\
\text { tos }\end{array}$ & - & - \\
\hline Sousa (2015) & $\begin{array}{l}\text { Medição I } \\
\text { Modelagem }\end{array}$ & $\begin{array}{l}\text { tempo de resposta, utilização de processador e } \\
\text { memória }\end{array}$ & Eucalyptus & MOODLE \\
\hline Bruschi et al. (2016) & Medição & Memória RAM, CPU, leitura em disco & Apache CloudStack & - \\
\hline Vogel et al. (2017) & Medição & Vazão, latência & Apache CloudStack & - \\
\hline Barros et al. (2017) & Medição & Latência e Consumo de energia & - & \\
\hline da Silva et al. (2019) & $\begin{array}{l}\text { Medição I } \\
\text { Modelagem }\end{array}$ & $\begin{array}{l}\text { Vazão, tempo de resposta, utilização de CPU, taxa } \\
\text { de escrita em HD }\end{array}$ & Apache CloudStack & MOODLE \\
\hline dos Santos et al. (2020) & Medição & Utilização de CPU, Memória RAM & ownCloud, NextCloud & - \\
\hline Este trabalho & Medição & $\begin{array}{l}\text { Memória, CPU, Consumo energia, vazão e tempo } \\
\text { de resposta }\end{array}$ & $\begin{array}{l}\text { Apache } \\
\text { CloudStack } \\
\text { e OpenStack }\end{array}$ & MOODLE \\
\hline
\end{tabular}

tante avaliar tais configurações.

Esses serviços compartilhados são divididos em: nuvem pública, privada, comunitária e híbrida,(Zhang et al., 2010).

- Nuvem pública: é aplicada quando sua finalidade é compartilhar serviços e recursos pela internet;

- Nuvem privada: é aplicada quando sua infraestrutura está totalmente (ou maior parte) dentro de uma estrutura privada, como serviços disponíveis que tenham a finalidade somente para uma única organização;

- Nuvem comunitária: a infraestrutura da nuvem é compartilhada por várias organizações.

- Nuvem híbrida: é aplicada quando sua infraestrutura está disponível agregando modelos de nuvens pública e privadas. Essas arquiteturas podem ser utilizadas de acordo com a finalidade de disponibilização de serviços, podendo utilizar apenas um dos modelos ou mais de um.

Assim sendo, para o gerenciamento desses serviços, plataformas de computação em nuvem como o Apache CloudStack (Apache, 2020), OpenNebula (Systems, 2020) e OpenStack (Vexxhost, 2020) foram criadas. A seguir, apresentamos mais informações sobre Apache CloudStack e, em seguida, sobre o OpenStack.

\subsubsection{Apache CloudStack}

O Apache CloudStack é um software de código aberto projetado para implantar e gerenciar amplas redes de máquinas virtuais com uma IaaS altamente escalável e disponível (Pauro, 2016). O Apache CloudStack gerencia a computação, a rede e os recursos de armazenamento. O CloudStack utiliza diversos hypervisors como KVM, vSphere e XenServer para virtualização e também tolera a API do AWS, além de suas próprias APIs (Kumar et al., 2014). Os recursos dentro da infraestrutura em nuvem do Apache CloudStack são denominados por: regiões, zonas, pods, clusters, hosts, armazenamento. (Apache, 2021).

- Regiões: uma coletânea de uma ou mais zonas geograficamente próximas gerenciadas por um ou mais servidores.

- Zonas: é análogo a um único datacenter. Uma zona consiste em uma ou mais pods e armazenamento secundário.

- Pods: um pod de regra é um rack ou fileira de racks que inclui um switch de camada 2 e um ou mais clusters.

- Clusters: um cluster incide em um ou mais hosts homogêneos e armazenamento primário.

- Hosts: um único nó de computação dentro de um cluster; muitas vezes um hypervisor.

- Armazenamento Primário: é uma solução de armazenamento fornecido a um único cluster para a execução real de imagens de disco da instância.

- Armazenamento Secundário: sendo um recurso de toda a zona que armazena imagens de disco (ex., imagens ISO).

\subsubsection{OpenStack}

O OpenStack é uma plataforma com conjunto crescente de soluções software colaborativa, criada pela NASA, para gerenciar e armazenar dados em uma infraestrutura virtualizada tanto em nuvem privada, como em pública. Esses serviços oferecidos pelo OpenStack permitem que juntos possam gerar a virtualização da computação, e a rede como Serviço (IaaS) formando um conjunto de serviços de software distribuídos interconectados.

O OpenStack apresenta as seguintes características: escalabilidade, flexibilidade, e é open source. Dessa forma, é possível redefinir os patamares de velocidade de implementação, custo e escalabilidade dos serviços, oferecendo certa flexibilidade em suas decisões tecnológicas. O OpenStack possui os seguintes componentes: Keystone (Serviço de Autenticação), Glance (serviço de imagem), Nova (serviço de computação), Neutron (serviço de rede), Horizon (serviço de painel-Dashboard), Cinder (bloco de armazenamento) e Swift (Objeto de armazenamento)(Vogel et al., 2016). 


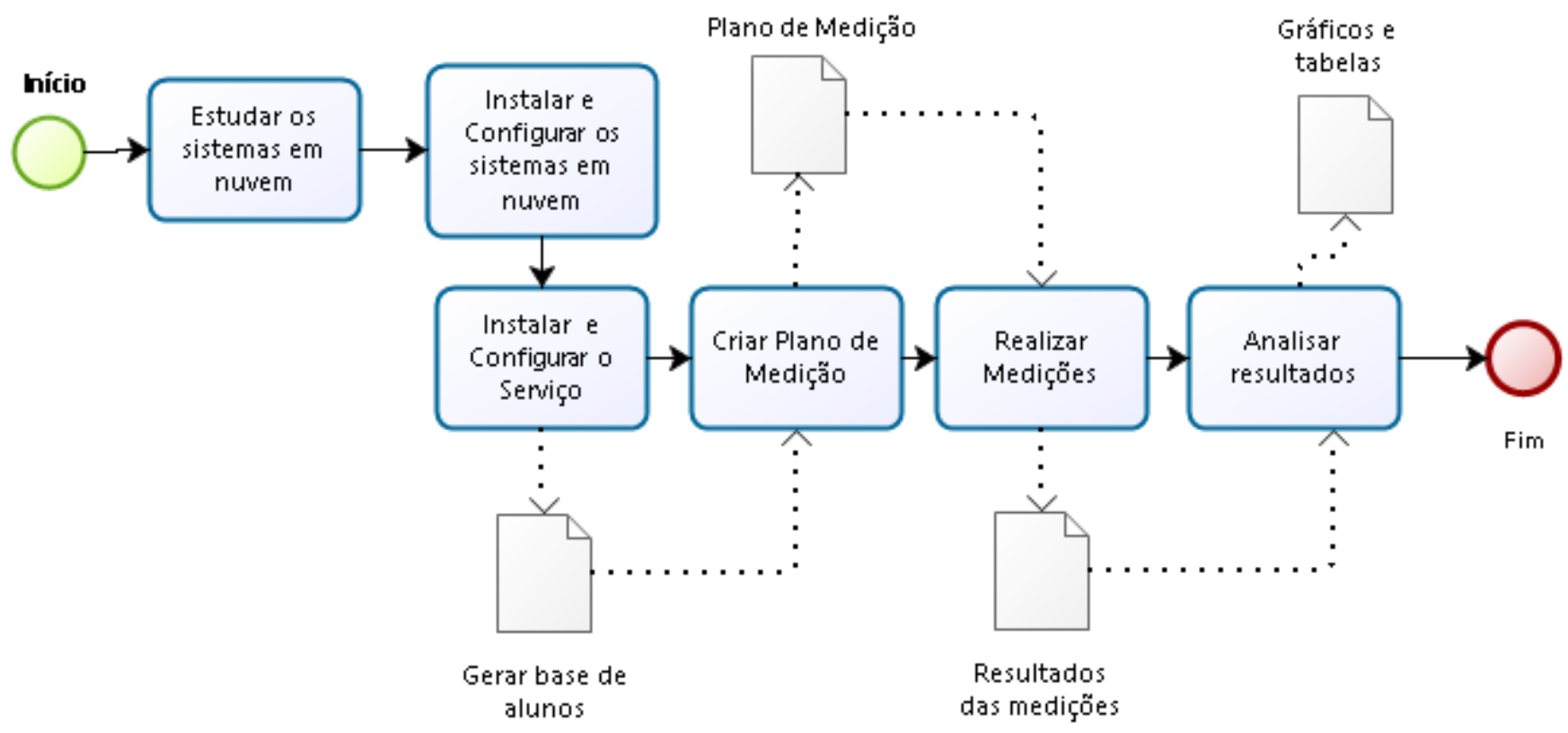

Figura 1: Metodologia adotada.

\subsection{Avaliação de Desempenho}

A avaliação de desempenho pode ser analisada via medições, modelagem e simulações (Callou et al., 2011). A mensuração de um sistema é o método mais direto para a avaliação de desempenho. As técnicas de medição apesar de fornecerem respostas exatas sobre o desempenho do sistema, não podem ser utilizadas (ou são muito caras) se um sistema estiver em operação. Já a modelagem analítica é utilizada para a abstração do sistema. Assim, o modelo deve conter detalhes do sistema que são essenciais para o seu comportamento. Por fim, a simulação do modelo poderá fornecer resultados não tão precisos quanto os fornecidos pela medição, mas é possível calcular as estimativas levando em consideração as precisões dos erros desejados (da Silva et al., 2019). Vale ressaltar que existem diversos tipos de modelos formais que podem ser utilizados para quantificar as métricas de desempenho desejadas. Neste trabalho, iremos ficar nas medições com a finalidade de se obter valores reais a fim de comparar tanto o desempenho como o consumo de energia.

\section{Metodologia}

Esta seção apresenta a metodologia utilizada para a avaliação de desempenho do AVA em nuvens privadas. Também são contextualizadas as atividades realizadas e os objetos gerados em cada uma das atividades dessa metodologia. A metodologia é ilustrada na Fig. 1. A seguir, cada atividade é detalhada.

Estudar os sistemas em nuvem: o propósito dessa fase é fazer um estudo com a finalidade de se obter uma compreensão da infraestrutura em nuvem a ser analisada e seus componentes. Além disso, nessa fase também devem ser analisados os serviços que serão configurados na nuvem (ex., Moodle) e as métricas que serão usadas para avaliação do desempenho.

Instalar e configurar os sistemas em nuvem: nesta atividade são realizadas a instalação e configuração da infraestrutura em nuvem (ex., Apache CloudStack e o OpenStack).

Instalar e configurar o serviço: esta fase relaciona-se a instalação e configuração de um serviço na máquina virtual de cada um dos sistemas em nuvem a serem comparados.

Criar o plano de medição: é criado um plano de medição na ferramenta Apache JMeter com o objetivo de realizar os experimentos nos ambientes. Além disso, nessa atividade ainda são criadas as bases de dados de alunos, professores, cursos, turmas e as atividades. Note que todos os dados inseridos são hipotéticos. O plano de medição é usado para definir as atividades a serem executadas como, por exemplo, acessar o Moodle, realizar login, acessar o curso, acessar a atividade, realizar upload de arquivos e sair do AVA. É nessa atividade que também devem ser definidas a quantidade de usuários e o tempo de inicialização para cada um.

Realizar medições: esta atividade corresponde as medições utilizando JMeter e/ou NMON, de acordo com o plano de medição criado na atividade anterior. Note que o Apache JMeter é responsável pela coleta de métricas como vazão e tempo de resposta, já o NMON pode ser utilizado para quantificar a utilização da CPU e da memória RAM. As medições devem ser repetidas, no mínimo, trinta vezes para se aumentar a confiabilidade nos resultados obtidos.

Analisar resultados: nesta etapa devem ser feitos os cálculos estatísticos da média, desvio padrão, e intervalo de confiança. E, ao final, são gerados gráficos de comparação dos resultados, como por exemplo, do tempo de resposta, vazão, utilização de $\mathrm{CPU}$, utilização da memória RAM, consumo de energia. 


\section{Estudo de Caso}

Essa seção apresenta um estudo para ilustrar a aplicabilidade da estratégia proposta. O estudo mostra um comparativo de desempenho e do consumo de energia entre os dois sistemas de nuvem, CloudStack e o OpenStack.

A Fig. 2 ilustra a visão geral do ambiente montado para a realização dos experimentos. $O$ ambiente é composto por três máquinas, sendo (i) uma cliente, com Apache JMeter para gerar a carga de trabalho e quantificar as métricas de desempenho; (ii) uma máquina com o ambiente de nuvem gerenciado pelo Apache CloudStack; e (iii) uma máquina com o ambiente de nuvem gerenciado pelo OpenStack. Em cada máquina com sistema de nuvem foi criada uma VM, configurado o Moodle e a ferramenta de medição NMON. É importante destacar que a comunicação entre essas máquinas é feita por um switch Gigabit. Além disso, pode-se observar a existência de um medidor para quantificar o consumo de energia das máquinas com o ambiente de nuvem.

Todas as máquinas utilizadas são equivalentes e são compostas por CPU Intel Core i5 de 3,4 GHz, $8 \mathrm{MB}$ Cache, 8GB RAM, NIC 1 Gigabit Ethernet 10/100/1000, 500 HD. O sistema operacional adotado foi o CentOS 7. Para ambas as nuvens foi instalado o hypervisor KVM. A VM utilizada possui processador com $2 \mathrm{GHz}$, com 2 core e $4 \mathrm{~GB}$ de memória RAM, 20GB de disco de armazenamento e sistema operacional Linux CentOS 7.

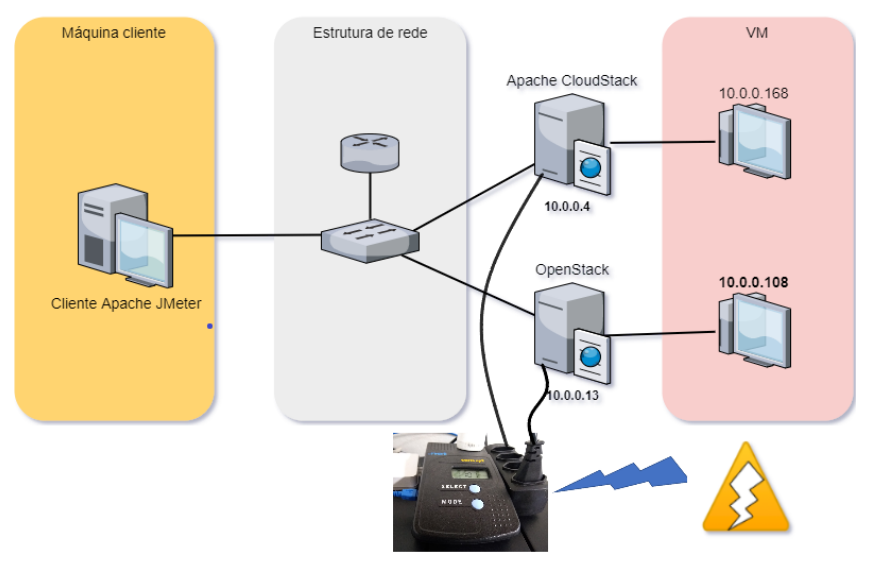

Figura 2: Infraestrutura montada para os experimentos.

O objetivo deste estudo de caso é comparar o desempenho e o consumo de energia entre o OpenStack e o Apache CloudStack. Para isso, cenários levando em consideração diferente número de clientes no sistema $(25,50,75$ e 100) foram adotados a fim de se fazer medições com a infraestrutura montada (ver Fig. 2). Em todos os cenários, o Moodle foi acessado para a realização das seguintes atividades: acessar o site AVA, logar no ambiente Moodle, acessar o curso, acessar uma atividade, selecionar um arquivo de $1 \mathrm{Mb}$, clique no arquivo de upload, upload do arquivo, salvar o envio e sair do AVA.

\subsection{Resultados consumo de energia}

A Fig. 3 ilustra o resultado comparativo do consumo de energia nas duas nuvens privadas (Apache CloudStack e OpenStack) para cada cenário analisado. Pode-se observar que o consumo de energia do OpenStack inicia inferior para o cenário com 25 clientes no sistema. No entanto, ao aumentar a quantidade de clientes, percebe-se que o consumo de energia do CloudStack passa a ser menor que o do OpenStack.

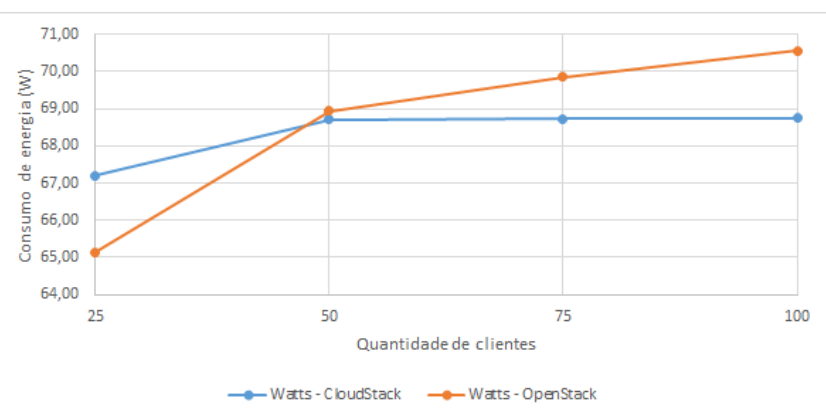

Figura 3: Consumo de energia (Watts)

Com a finalidade de se comprovar que a diferença do consumo de energia dos dois ambientes de nuvem privada é significativo, aplicou-se o Teste T Pareado (Montgomery and Runger, 2010). A Tabela 2 apresenta o resumo estatístico desse teste. Neste comparativo, o valor de $\mathrm{P}$ ficou em 0,001 . Dessa forma, como o valor de P ficou inferior ao $\alpha$ adotado $(0,05)$, pode-se afirmar que as duas amostras são estatisticamente distintas com $95 \%$ de confiança. Logo, o CloudStack possui o consumo de energia diferente do OpenStack.

Tabela 2: Teste-t: duas amostras em par para médias (W).

\begin{tabular}{ll}
\hline Estatística & Resultado \\
\hline Quantidade (Número) & 30 \\
Desvio Padrão & 1,008407188 \\
Nível de Confiança & $95 \%$ \\
Margem de Erro & 0,361 \\
Limite Inferior & $-2,7457$ \\
Limite Superior & $-1,2956$ \\
Valor de P & 0,001 \\
\hline
\end{tabular}

A Tabela 3 mostra em maiores detalhes os resultados da medição do consumo de energia de cada computador com o sistema de nuvem Apache CloudStack e OpenStack. Nessa tabela, a coluna $Q C$ representa os cenários com diferente quantidade de clientes analisados. Na tabela, são apresentados ainda a diferença em percentual no consumo energético de cada sistema em nuvem para cada cenário analisado. Além disso, pode-se perceber que o consumo médio do OpenStack $(69,73 \mathrm{~W})$ é superior ao do CloudStack $(68,72 \mathrm{~W})$. Essa diferença no consumo de energia pode, aparentemente, parecer pequena. No entanto, ao se con- 
siderar um data center que faz uso de dezenas de racks, onde cada rack é composto por vários servidores, uma redução no consumo de energia de cada servidor passa a ser representativa.

Tabela 3: Comparação do consumo de energia OpenStack vs CloudStack.

\begin{tabular}{cccc}
\hline QC & CloudStack(W) & OpenStack(W) & Diferença(\%) \\
\hline 25 & 67,20 & 65,14 & $-3,16 \%$ \\
50 & 68,71 & 68,93 & $0,32 \%$ \\
75 & 68,72 & 69,85 & $1,62 \%$ \\
100 & 68,74 & 70,55 & $2,57 \%$ \\
\hline \hline \multicolumn{4}{c}{$(30$ amostras) } \\
Médias & 68,72 & 69,73 & $1,45 \%$ \\
Desv. Pad. & 0,600 & 0,914 & \\
\hline
\end{tabular}

\subsection{Resultados utilização da CPU}

A Fig. 4 mostra os resultados da utilização da CPU (ou seja, da VM) em cada nuvem privada. Pode-se observar que a utilização da CPU do CloudStack inicia superior (cenários com 25 e 50 clientes). No entanto, com o aumento dos clientes no sistema, observou-se que o nível de utilização passou a se inverter, mas com diferença mínima.

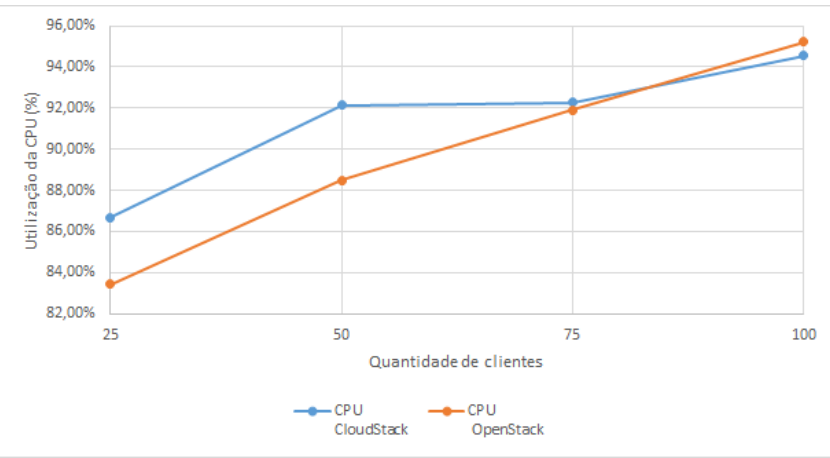

Figura 4: Utilização da CPU (\%)

Similarmente ao estudo do consumo de energia, também foi aplicado o Teste T Pareado. A Tabela 4 apresenta o resultado do resumo estatístico desse teste. Neste comparativo, o valor de $P$ ficou em 0,001 . Sendo assim, existe diferença significativa, com $95 \%$ de confiança, entre os resultados dos dois ambientes analisados.

A Tabela 5 mostra em maiores detalhes os resultados da medição do nível de utilização da CPU da VM para cada cenário considerando o Apache CloudStack e o OpenStack. Destaca-se que o nível de utilização médio da CPU obtido para o OpenStack foi superior (95,2\% vs $93,5 \%)$. Embora a diferença possa parecer pequena, assumindo cenários mais complexos onde são adotados servidores de grande porte, que hospedam um conjunto de VMs, essa diferença representa uma redução nos recursos adotados por cada VM e, assim, essa redução no uso de CPU passa a ser representativa para o servidor.
Tabela 4: Teste-t: duas amostras em par para médias (CPU).

\begin{tabular}{ll}
\hline Estatística & Resultado \\
\hline Quantidade (Número) & 30 \\
Desvio Padrão & 1,026774 \\
Nível de Confiança & $95 \%$ \\
Margem de Erro & 0,010 \\
Limite Inferior & $-0,027$ \\
Limite Superior & $-0,0071$ \\
Valor de P & 0,001 \\
\hline
\end{tabular}

Tabela 5: Comparação do nível de utilização da CPU OpenStack vs CloudStack.

\begin{tabular}{cccc}
\hline QC & CloudStack & OpenStack & Diferença(\%) \\
\hline 25 & $86,67 \%$ & $83,45 \%$ & $-3,86 \%$ \\
50 & $92,13 \%$ & $88,49 \%$ & $-4,11 \%$ \\
75 & $92,26 \%$ & $91,91 \%$ & $-0,38 \%$ \\
100 & $94,55 \%$ & $95,21 \%$ & $0,69 \%$ \\
\hline \hline \multicolumn{4}{c}{$(30$ amostras) } \\
Média & $93,5 \%$ & $95,2 \%$ & $1,79 \%$ \\
Desv.Pad. & 0,019 & 0,020 & \\
\hline
\end{tabular}

\subsection{Resultados do consumo de memória RAM}

A Fig. 5 mostra um gráfico comparativo com o consumo de memória RAM para cada nuvem e contemplando os cenários analisados. O OpenStack, nos cenários de até 75 clientes, apresenta um consumo de memória menor quando comparado ao CloudStack. No entanto, com 100 clientes, esse cenário se inverteu. 0 OpenStack passou a demandar mais memória RAM para realizar as mesmas atividades.

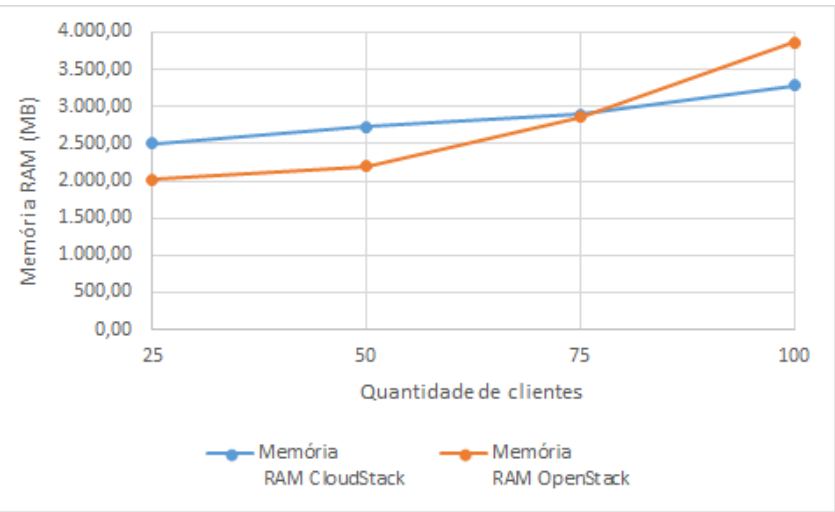

Figura 5: Comparativo do consumo de memória RAM.

A Tabela 6 mostra em maiores detalhes os resultados da medição do consumo de memória RAM de cada nó com o sistema de nuvem Apache CloudStack e OpenStack, apresentando o percentual da diferença na utilização da memória RAM das máquinas virtuais. Todavia, pode-se perceber que na média, para os cenários analisados, o Apache CloudStack teve um consumo de memória RAM mais alto. É possível observar uma inversão no consumo de recur- 
sos, tanto em relação ao nível de utilização da CPU (??), como também em relação ao consumo de memória RAM, onde observou-se que o OpenStack apresenta um comportamento superior quando o sistema ainda não se encontra com uma carga de estresse elevada (ex., 25 e 50 clientes), consumindo assim menos recursos computacionais. No entanto, quando se aumenta a quantidade de clientes simultâneos no sistema, observou-se que esse comportamento foi alterado. Essa alteração pode ter ocorrido em virtude das diferenças no gerenciamento dos recursos a partir de cada um desses ambientes e, assim, o ambiente CloudStack consegue gerenciar melhor os recursos computacionais quando sob uma forte carga de trabalho.

Tabela 6: Comparação do consumo de memória RAM OpenStack vs Apache CloudStack.

\begin{tabular}{cccc}
\hline QC & CloudStack(MB) & OpenStack(MB) & Diferença(\%) \\
\hline 25 & 2506,75 & 2015,23 & $-24,39 \%$ \\
50 & 2732,03 & 2197,91 & $-24,30 \%$ \\
75 & 2894,64 & 2855,32 & $-1,38 \%$ \\
100 & 3282,53 & 3860,89 & $14,98 \%$ \\
\hline \hline \multicolumn{4}{c}{$(30$ amostras) } \\
Média & 2854,00 & 2732,30 & $-4,45 \%$ \\
Desv.Pad. & 326,98 & 834,39 & \\
\hline
\end{tabular}

Similar aos resultados anteriores, também foi utilizado o teste T Pareado. A Tabela 7 apresenta o resumo estatístico desse teste, onde percebe-se que as duas amostras dos resultados são distintas com 95\% de confiança.

Tabela 7: Teste-t: duas amostras em par para médias Memória RAM da VW.

\begin{tabular}{ll}
\hline Estatística & Resultado \\
\hline Quantidade (Número) & 30 \\
Desvio Padrão & 1,10782 \\
Nível de Confiança & $95 \%$ \\
Margem de Erro & 0,039 \\
Limite Inferior & $-0,181$ \\
Limite Superior & $-0,099$ \\
Valor de P & 0,001 \\
\hline
\end{tabular}

\subsection{Resultados para a Vazão}

A Fig. 6 mostra o resultado da comparação da vazão. Podese perceber que ambas as nuvens privadas apresentaram uma vazão praticamente constante (desvio padrão inferior a 0,08) para os cenários analisados com 25, 50, 75 e 100 clientes. No entanto, existe uma diferença significativa entre cada uma das duas nuvens privadas, onde o CloudStack apresentou uma capacidade média de vazão em 4,4 clientes atendidos por segundo enquanto que para o OpenStack se observou uma média de 2,76.

A Tabela 8 mostra em maiores detalhes os resultados da medição da vazão de cada nó com o sistema de nuvem Apache CloudStack e OpenStack, apresentando o percentual da diferença na utilização da vazão das máquinas virtuais.

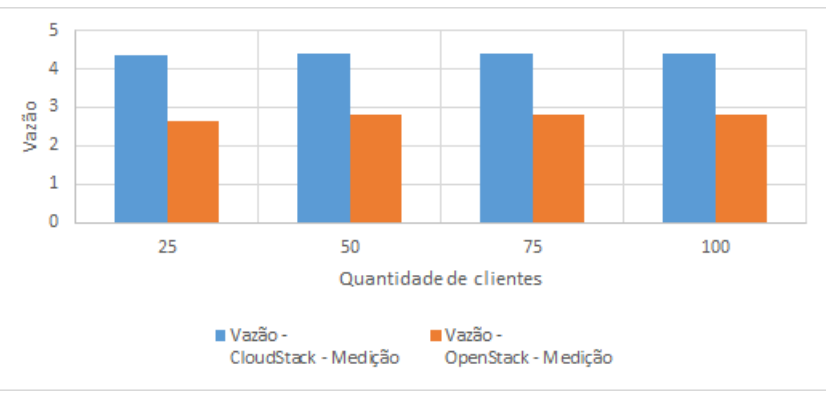

Figura 6: Comparação da vazão

Tabela 8: Comparação vazão - Apache CloudStack vs OpenStack.

\begin{tabular}{cccc}
\hline QC & CloudStack(c/s) & OpenStack(c/s) & Diferença(\%) \\
\hline 25 & 4,37 & 2,65 & $-65,91 \%$ \\
50 & 4,40 & 2,79 & $-58,71 \%$ \\
75 & 4,40 & 2,80 & $-57,14 \%$ \\
100 & 4,41 & 2,81 & $-57,94 \%$ \\
\hline \hline \multicolumn{4}{c}{$(30$ amostras) } \\
Média & 4,395 & 2,762 & $-59,12 \%$ \\
Desv.Pad. & 0,017 & 0,075 & \\
\hline
\end{tabular}

Assumindo o cenário com 25 clientes, o CloudStack apresentou uma vazão bem superior em relação ao OpenStack (65\%). É importante destacar que esse melhor desempenho do CloudStack permaneceu para os demais cenários analisados. No entanto, à medida que se aumenta a quantidade de clientes no sistema, o OpenStack obteve uma melhor vazão. No entanto, mesmo assim, permaneceu inferior em relação ao CloudStack com apenas 57,9\% assumindo o cenário com 100 clientes, conforme mostrado na Tabela 8 .

\subsection{Resultados para o Tempo de Resposta}

A Fig. 7 mostra o resultado da comparação do tempo de resposta das duas VMs. O CloudStack apresentou sempre um tempo de resposta melhor do que o OpenStack em todos os cenários analisados. Os resultados obtidos para o tempo de resposta obedeceram a um aumento praticamente constante em relação ao incremento de clientes no sistema. Por exemplo, assumindo os cenários com o OpenStack, pode-se perceber a partir da Tabela 9 que no cenário com 25 clientes o tempo de resposta foi de 104,94 segundos, e ao se dobrar a quantidade de clientes, esse tempo praticamente dobrou, indo para 210,34 segundos. Considerando 100 clientes, também foi possível observar que o tempo de resposta saiu de 210,34 segundos (cenário com 50 clientes) para 412,78 segundos. Um comportamento similar foi observado ao se utilizar o CloudStack.

A Tabela 9 apresenta com mais detalhes os resultados da medição obtidos para o tempo de resposta dos sistemas em nuvem Apache CloudStack e OpenStack. O Tempo de resposta da VM com OpenStack apresenta uma significativa diferença, para 25 clientes por exemplo, um percentual de $51,1 \%$ maior em relação ao mesmo cenário para o Apache CloudStack. Em resumo, o Apache CloudStack apresentou 


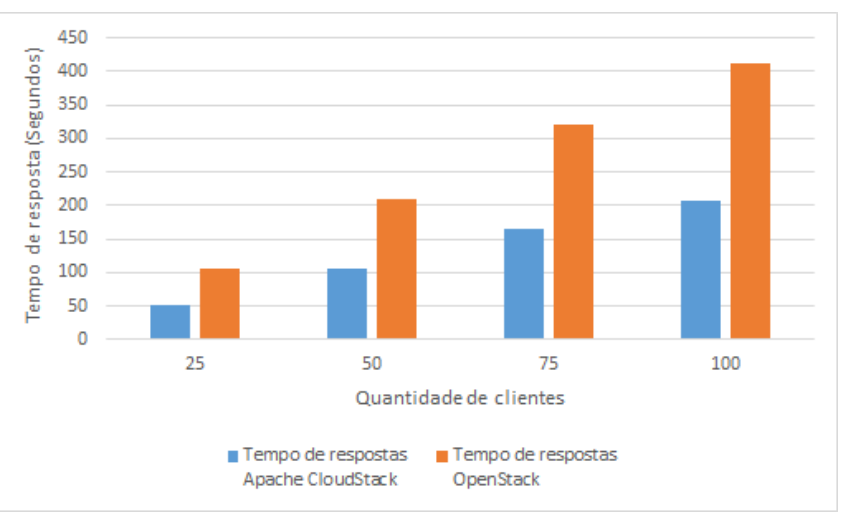

Figura 7: Comparação do Tempo de resposta

um tempo de resposta menor em todas as medições, como média de 132,40 segundos. O OpenStack, por sua vez, teve um tempo médio de resposta de 261,95 segundos.

Tabela 9: Comparação do tempo de resposta Apache CloudStack vs OpenStack.

\begin{tabular}{cccc}
\hline QC & CloudStack & OpenStack & Diferença(\%) \\
\hline 25 & 51,32 & 104,94 & $51,10 \%$ \\
50 & 106,86 & 210,34 & $49,20 \%$ \\
75 & 165,46 & 319,76 & $48,25 \%$ \\
100 & 205,95 & 412,78 & $50,11 \%$ \\
\hline \hline \multicolumn{4}{c}{$(30$ amostras) } \\
Média & 132,40 & 261,95 & $49,46 \%$ \\
Desv.Pad. & 67,64 & 133,43 & \\
\hline
\end{tabular}

De uma forma geral, é possível observar que referente ao consumo de energia entre as duas nuvens, o Apache CloudStack obteve um menor consumo quando utilizamos a quantidade máxima de clientes simultaneamente no AVA. Já a utilização da CPU da Máquina Virtual para uma quantidade inicial de usuários, o OpenStack obteve melhor desempenho. Porém, à medida que expandimos a quantidade de clientes, o Apache CloudStack chega a uma utilização 2,57\% menor. Para a Memória RAM, o OpenStack nas primeiras quantidades de clientes demonstrou uma menor utilização desse recurso. No entanto, à medida em que se aumentou a quantidade de requisições, o Apache CloudStack obteve melhor desempenho. Já em relação a vazão, foi revelado que o Apache CloudStack tem a vazão média duas vezes maior quando comparado com o OpenStack. Por fim, o tempo de resposta do Apache CloudStack possui um melhor desempenho.

\section{Conclusão}

Este artigo analisou o consumo de energia e o desempenho do ambiente virtual de aprendizagem Moodle hospedado nas nuvens privadas Apache CloudStack e OpenStack. Cenários com diferentes capacidades de carga foram analisados para os dois sistemas, onde, através da metodologia proposta, se pode realizar tal estudo comparativo.

Os resultados revelaram que o consumo de energia do
OpenStack foi em média 1,5\% maior que o do CloudStack. Esse comportamento pode ser explicado pelo fato de que o OpenStack usa mais CPU ( $1,8 \%$ em média a mais do que o CloudStack). Além disso, é possível observar que o OpenStack demandou um maior tempo de resposta (praticamente $100 \%$ superior), e isso pode ter ocorrido pelo fato de que ele apresentou uma vazão bem inferior (aproximadamente $60 \%$ da vazão do CloudStack). E, assim, o CloudStack acaba por demandar um menor consumo de energia para os cenários analisados. Como trabalho futuro, pretende-se propor modelos para representar o comportamento desses sistemas e, assim, poder estimar o comportamento desses sistemas em cenários mais complexos.

\section{Agradecimentos}

O presente trabalho foi realizado com apoio do CNPq, FACEPE, CAPES e da UFRPE.

\section{Referências}

Apache (2020). Apache cloudstack - open source cloud computing. Disponível em http://cloudstack. apache . org/.

Apache (2021). Apache cloudstack - open source cloud computing. Disponível em http://docs.cloudstack. apache.org/en/latest/conceptsandterminology/ concepts.html\#cloudstack-terminology.

Barros, J., Callou, G. and Gonçalves, G. (2017). Análise integrada de desempenho e consumo de energia em sistemas de armazenamento de dados distribuídos, Anais do XV Workshop em Clouds e Aplicações, SBC. Available at https://sol.sbc.org.br/index.php/wcga/ article/view/2551.

Bruschi, G. C., Spolon, R., Pauro, L. L., Lobato, R. S., Manacero, A. and Cavenaghi, M. A. (2016). Stackact: Performance evaluation in an iaas cloud multilayer, 15th International Symposium on Parallel and Distributed Computing (ISPDC), IEEE, pp. 149-156. https://doi .org/10. 1109/ISPDC. 2016.27.

Callou, G., Maciel, P., Tavares, E., Andrade, E., Nogueira, B., Araujo, C. and Cunha, P. (2011). Energy consumption and execution time estimation of embedded system applications, Microprocessors and Microsystems 35(4): 426440. https://doi.org/10.1016/j.micpro.2010.08.006.

da Silva, A. V. G., de Lima, C. J. and de Almeida Callou, G. R. (2019). Análise de desempenho do ambiente virtual de aprendizagem na nuvem privada apache cloudstack, GESTÃO. Org 17(8): 120-133. http://dx.doi.org/ 10.21714/1679-18272019v17Esp.p120-133.

dos Santos, D. M. L., Vale, K. M. A. C. and de Alencar, F. M. R. (2020). Avaliação de desempenho de nuvens privadas: um comparativo entre owncloud, nextcloud e pydio, Brazilian Journal of Development 6(6): 40549-40566. https://doi.org/10.34117/bjdv6n6-553. 
FÉ, I. d. S. (2017). Planejamento de transcodificação de vídeo em nuvem elástica, Master's thesis, Universidade Federal de Pernambuco. Disponível em https://repositorio. ufpe.br/handle/123456789/25075.

Feitelson, D. G. (2015). Workload modeling for computer systems performance evaluation, Cambridge University Press. https://doi.org/10.1017/CB09781139939690.

Kim, D. S., Machida, F. and Trivedi, K. S. (2009). Availability modeling and analysis of a virtualized system, 2009 15th IEEE Pacific Rim International Symposium on Dependable Computing, pp. 365-371. http://dx.doi.org/10. 1109/PRDC.2009.64.

Kumar, R., Jain, K., Maharwal, H., Jain, N. and Dadhich, A. (2014). Apache cloudstack: Open source infrastructure as a service cloud computing platform, Proceedings of the International Journal of advancement in Engineering technology, Management and Applied Science pp. 111-116. Disponível em http: //ijrcemas. com/wp-content/uploads / 2014/08/IJB024.pdf.

Machida, F., Andrade, E., Kim, D. S. and Trivedi, K. S. (2011). Candy: Component-based availability modeling framework for cloud service management using sysml, 2011 IEEE 3oth International Symposium on Reliable Distributed Systems, IEEE, pp. 209-218. http: //dx.doi.org/10.1109/SRDS.2011.33.

Montgomery, D. C. and Runger, G. C. (2010). Applied statistics and probability for engineers, John Wiley \& Sons. Disponível em

NIST (2014). Nist cloud computing program., http://www.nsti.gov/itl/cloud/index.cfm. Disponível em http://www.nsti.gov/itl/cloud/index.cfm.

Pauro, L. L. (2016). Auditoria e monitoramento de eventos inconsistentes em instâncias de máquinas virtuais em iaas no orquestrador apache cloudstack. Disponível em https://repositorio.unesp.br/handle/11449/147090.

Sousa, E., Lins, F., Tavares, E. and Maciel, P. (2017). Cloud infrastructure planning considering different redundancy mechanisms, Computing 99(9): 841-864. https : //doi.org/10.1007/s00607-016-0533-6.

Sousa, E. T. G. d. (2015). Modelagem de desempenho, dependabilidade e custo para o planejamento de infraestruturas de nuvens privadas. Disponível em https: //repositorio.ufpe.br/handle/123456789/15258.

Systems, O. (2020). Opennebula - the open source cloud management platform. Disponível em https: //opennebula.io/.

Torres, E., Callou, G., Alves, G., Accioly, J. and Gustavo, H. (2016). Storage services in private clouds: Analysis, performance and availability modeling, 2016 IEEE International Conference on Systems, Man, and Cybernetics (SMC), pp. 003288-003293. https://doi.org/10.1109/ SMC. 2016.7844741.

Vexxhost (2020). Openstack. Disponível em https : //www . openstack.org/.
Vogel, A., Griebler, D., Maron, C. A. F., Schepke, C. and Fernandes, L. G. (2016). Private iaas clouds: A comparative analysis of opennebula, cloudstack and openstack, 2016 24th Euromicro International Conference on Parallel, Distributed, and Network-Based Processing (PDP), pp. 672679. http://dx.doi.org/10.1109/PDP.2016.75.

Vogel, A., Griebler, D., Schepke, C. and Fernandes, L. G. (2017). An intra-cloud networking performance evaluation on cloudstack environment, 201725th Euromicro International Conference on Parallel, Distributed and Network-based Processing (PDP), IEEE, pp. 468-472. https://doi.org/10.1109/PDP.2017.40.

Zhang, Q., Cheng, L. and Boutaba, R. (2010). Cloud computing: state-of-the-art and research challenges, Journal of internet services and applications 1(1): 7-18. https: //doi.org/10.1007/s13174-010-0007-6.

Zota, R. and Petre, I. A. (2014). An overview of the most important reference architectures for cloud computing., Informatica Economica 18(4). https : //doi .org/10.12948/ issn14531305/18.4.2014.03. 